\title{
Prüfungsrecht: Die Begründung von Prüfungsentscheiden
}

Im Prüfungsrecht ist die Begründung der Leistungsbewertung von entscheidender Bedeutung. Erst die Begründung erlaubt es dem Prüfling und der Beschwerdeinstanz die Bewertung nachzuvollziehen. Art. 29 Abs. 2 BV stellt die Mindestgarantie bezüglich Anforderungen an die Begründung dar. Grosse Teile der Rechtsprechung erachten dabei die schlichte Notenbekanntgabe im Verwaltungsverfahren als ausreichend. Gemäss dieser Praxis reicht es aus, wenn die nachvollziehbare Begründung im Beschwerdeverfahren nachgeschoben wird und der Prüfling in einem zweiten Schriftenwechsel dazu Stellung nehmen kann. Der Autor setzt sich sowohl mit den Anforderungen an die Begründung als auch mit den Rechtsfolgen einer verletzten Begründungspflicht auseinander. Zudem versucht er aufzuzeigen, weshalb von Prüfungsbehörden im Verwaltungsverfahren zumindest eine summarische Begründung verlangt werden muss. Bei mündlichen und praktischen Prüfungen sollte die Rechtsprechung sodann eine Aufzeichnungs-/Protokollierungspflicht aus Art. 29 Abs. 2 BV ableiten.

\section{Einleitung}

II. Lehre und Rechtsprechung

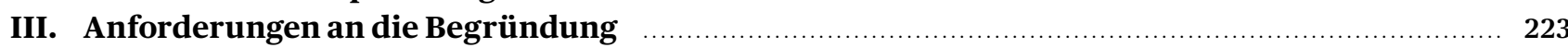

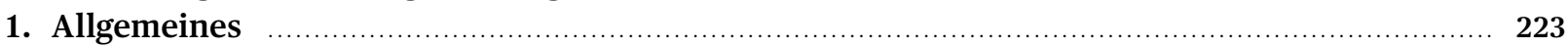

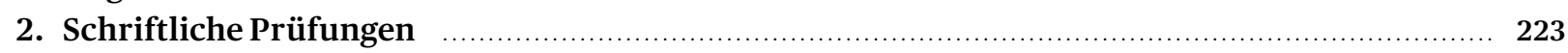

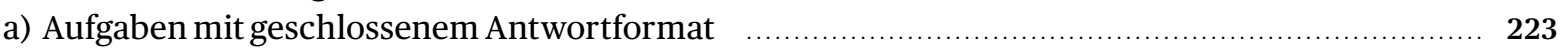

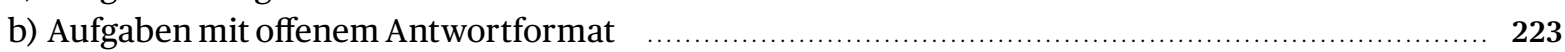

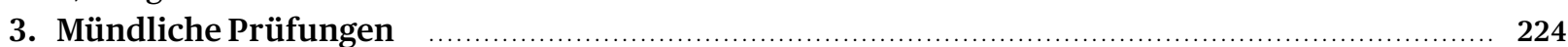

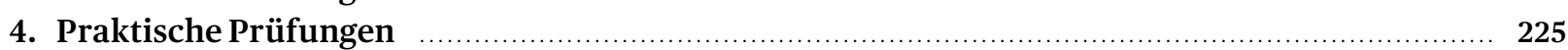

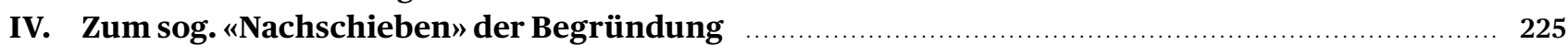

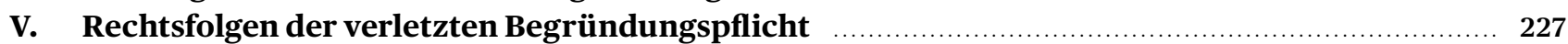

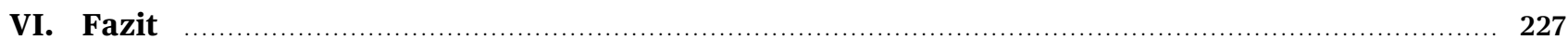

Zitiervorschlag:

RAFAEL ZÜND, Prüfungsrecht: Die Begründung

von Prüfungsentscheiden, sui generis 2021, S. 219

Rafael Zünd, MLaw und BSc in Psychology, Anwaltspraktikant, zurzeit bei der advocomplex gmbh in Bern, zuvor bei der Erziehungsdirektion des Kantons Bern [heute: Bildungs- und Kulturdirektion] (leafar@ihost.ch). In diesem Artikel wird der Einfachheit halber teilweise die weibliche und teilweise die männliche Form verwendet. Die nicht erwähnten Geschlechtsformen sind jeweils mitgedacht.

URL: sui-generis.ch/185

DOI: https://doi.org/10.21257/sg.185

Dieses Werk ist lizenziert unter einer Creative Commons Namensnennung - Weitergabe unter gleichen Bedingungen 4.o International Lizenz. 


\section{Einleitung}

1 Die Verfassungsnorm Art. 29 Abs. 2 BV1 garantiert die Mindestanforderungen an das rechtliche Gehör. ${ }^{2}$ Das rechtliche Gehör stellt sowohl ein persönlichkeitsbezogenes Mitwirkungsrecht als auch ein Mittel zur Sachverhaltsabklärung dar. ${ }^{3}$ Aus diesem Verfahrensrecht ergibt sich insbesondere die Pflicht der Behörde, ihren Entscheid zu begründen. Die Begründung muss derart abgefasst sein, dass die betroffene Person nachvollziehen kann, weshalb die Behörde auf diese Weise entschieden hat. Sodann muss die betroffene Person in der Lage sein, den Entscheid sachgerecht anfechten zu können, und die Beschwerdeinstanz muss den Entscheid anhand der Begründung nachvollziehen können. ${ }^{4}$ Dabei muss die Begründungsdichte umso höher sein, je schwerer der Entscheid in die Rechtsstellung der betroffenen Person eingreift und je grösser der Ermessensspielraum der Behörde ist. ${ }^{5}$ Eine nachvollziehbare Begründung vermittelt der betroffenen Person das Gefühl, von der Behörde ernst genommen zu werden. ${ }^{6}$ Eine fehlende oder ungenügende bzw. nicht nachvollziehbare Begründung stösst demgegenüber auf Unverständnis und verletzt das rechtliche Gehör gemäss Art. 29 Abs. 2 BV.7

2 Der Begründungspflicht kommt im Prüfungsrecht eine zentrale Bedeutung zu. Das liegt einerseits am enormen Ermessensspielraum der Prüfungsbehörden. Infolgedessen nehmen Beschwerdeinstanzen bei Prüfungsentscheiden grundsätzlich lediglich eine beschränkte Über-

1 Bundesverfassung der Schweizerischen Eidgenossenschaft vom 18. April 1999 (BV; SR 101).

2 PIERRE TSCHANNEN/ULRICH ZIMMERLI/ MARKUS MÜLLER, Allgemeines Verwaltungsrecht, 4. Aufl., Bern 2014, S. 289f.; ULRICH HÄFELIN/ GEORG MÜLLER / FELIX UHLMANN, Allgemeines Verwaltungsrecht, 8. Aufl., Zürich/St. Gallen 2020, Rz.1003.

3 Vgl.Urteil des Bundesgerichts 2C_1004/2017 vom 29. Mai 2018E.3.1; HÄFELIN/MÜLLER/UHLMANN (Fn. 2), Rz.1001; LORENZ KNEUBÜHLER, Gehörsverletzung und Heilung, ZBI 99/1998, S. 100; REGINA KIENER/ BERNHARD RÜTSCHE / MATHIAS KUHN, Öffentliches Verfahrensrecht, 2. Aufl., Zürich / St. Gallen 2015, Rz. 229; REGINA KIENER / WALTER KäLIN / JUDITH WYTTENBACH, Grundrechte, 3. Aufl., Bern 2018, S. 512, sowie PATRICK SUTTER, VwVG-Kommentar, 2. Aufl., Zürich/ St. Gallen 2019, Art. 29 Rz. 8.

4 Urteil des Bundesgerichts 2C_505/2019 vom 13. September 2019 E. 4.2.1; Urteil des Bundesgerichts 2D_10/2019 vom 6. August 2019 E. 4.2; KIENER/RÜTSCHE/KUHN (Fn. 3), Rz.243f., HÄFELIN/MÜLLER/UHLMANN (Fn. 2), Rz.1070 f. SOwie NORBERT NIEHUES / EDGAR FISCHER / CHRISTOPH JEREMIAS, Prüfungsrecht, 7. Aufl., München 2018, Rz.706.

5 TSCHANNEN/ZIMMERLI/MÜLLER (Fn. 2), S. 272 f.; KIENER/KÄLIN/ WYTTENBACH (Fn. 3), S. 522; HÄFELIN/MÜLLER/UHLMANN (Fn. 2), Rz. 411 und 1072; RENÉ WIEDERKEHR, Die Begründungspflicht nach Art. 29 Abs. 2 BV und die Heilung bei Verletzung, ZBI 111/2010, S. 484 und 487; sowie MARTIN AUBERT, Bildungsrechtliche Leistungsbeurteilungen im Verwaltungsprozess, Diss. Bern 1997, S.144f.

6 MARKUS MÜLLER, Psychologie im öffentlichen Verfahren, Bern 2010, S. 45; AUBERT (Fn. 5), S. 140.

7 TSCHANNEN/ZIMMERLI/MÜLLER (Fn. 2), S. 273. prüfung vor. ${ }^{8}$ Das Verwaltungsgericht des Kantons Bern beschreibt dieses Vorgehen jeweils wie folgt: «Soweit es um die Beurteilung von Prüfungsleistungen geht, auferlegt es [das Verwaltungsgericht] sich im Rahmen der Rechtskontrolle praxisgemäss eine gewisse Zurückhaltung, weil es wesentliche Sachumstände nicht genügend namhaft machen kann, um sie gleich kompetent zu würdigen wie die verfügende Instanz.» ${ }^{9}$ Selbst wenn Beschwerdebehörden gemäss Verfahrenserlass zu einer Angemessenheitskontrolle verpflichtet sind, auferlegen sie sich eine grosse Zurückhaltung und beschränken sich im Wesentlichen auf eine grobe Prüfung der Nachvollziehbarkeit. ${ }^{10}$ Andererseits handelt es sich bei Prüfungsentscheiden oft um subjektive Bewertungen menschlichen Verhaltens, welche durch eine nachvollziehbare Begründung objektiviert werden sollen. ${ }^{11}$ Demnach sollten im Prüfungsrecht eher hohe Anforderungen an die Begründung gestellt werden.

\section{Lehre und Rechtsprechung}

Die schweizerische Rechtslehre macht die Anforderun- 3 gen an die Begründung von Verfügungen allgemein abhängig vom konkreten Einzelfall. Demnach werden bei komplexen Sach- und Rechtslagen, schweren Eingriffen und grossem Ermessen der Prüfungsbehörde erhöhte Anforderungen an die Begründung gestellt. Bei klarer Sach- oder Rechtslage sowie bei Massenverfügungen gelten demgegenüber tiefere Anforderungen. ${ }^{12}$ Bezüglich Prüfungsrecht vertritt WIEDERKEHR die Auffassung, dass aus der Begründung zumindest hervorgehen muss, welche Antworten korrekt waren, wo Mängel festgestellt wurden, welches die richtigen Antworten gewesen wären und wie die Mängel gewichtet wurden. ${ }^{13}$ AUBERT erachtet sodann gewisse bildungsrechtliche Leistungsbeurteilungen zumindest als einem Massenverfahren ähnlich, weshalb in solchen Fällen summarische Begründungen genügen können. ${ }^{14}$

8 Vgl. dazu Urteil des Bundesgerichts 2C_212/2020 vom 17. August 2020 E.3.2.

9 Vgl. z.B. Urteil des Verwaltungsgerichts des Kantons Bern 100.2016.181U vom 16. Februar 2017 E.1.2.

10 Vgl. dazu etwa das Urteil des Bundesverwaltungsgerichts B-6261/2008 vom 4. Februar 2010 E. 4.1; den Zwischenentscheid der Erziehungsdirektion des Kantons Bern 4800.600.350.90/16 (758580) vom 17. Oktober 2017 E. 2.1, zusammengefasst in BVR 2018 S.128f.; AUBERT (Fn. 5), S. $115 f$.

11 Vgl. AUBERT (Fn. 5), S. 142

12 AUBERT(Fn. 5), S.144f.; HÄFELIN/MÜLLER/UHLMANN (Fn. 2), Rz.1072; KIENER/KÄLIN/WYTTENBACH (Fn. 3), S. 522; TSCHANNEN/ZIMMERLI/ MÜLLER (Fn. 2), S. 272 f.; WIEDERKEHR (Fn. 5), S. 484.

13 WIEDERKEHR (Fn. 5), S. 490.

14 AUBERT (Fn. 5), S. 146. 
4 In der deutschen Literatur wird die Ansicht vertreten, dass nicht der Umfang der Prüfungsbegründung entscheidend ist, sondern ob die Begründung die konkrete Bewertung zu rechtfertigen vermag. Die Prüfungsbegründung muss sich dieser Ansicht nach zumindest zu den für das Ergebnis massgebenden Punkten äussern. ${ }^{15}$ Sodann werden an die Begründung bei schriftlichen Prüfungen höhere Anforderungen gestellt als bei mündlichen Prüfungen. Bewertungen schriftlicher Prüfungen sind nach dieser Auffassung stets schriftlich zu begründen, wogegen solche mündlicher Prüfungen in einem ersten Schritt auch nur mündlich begründet werden können. Weiter wird in der deutschen Lehre die Auffassung vertreten, wonach bei mündlichen Prüfungen die Begründung ganz ausbleiben kann, wenn der Prüfling keine solche verlangt. ${ }^{16}$ Schliesslich wird in der deutschen Literatur die Ansicht vertreten, wonach eine fehlerhafte oder unvollständige Begründung durch die Prüfungsbehörde im Verlauf des Beschwerdeverfahrens korrigiert werden kann. ${ }^{17}$

5 Auch das Bundesgericht lässt ein solches «Nachschieben» der Prüfungsbegründung im Beschwerdeverfahren gemäss ständiger Praxis grundsätzlich zu. Insbesondere in den älteren Entscheiden hielt das Bundesgericht meistens fest, dass der Anspruch auf rechtliches Gehör gemäss Art. 29 Abs. 2 BV nicht schon dann verletzt sei, wenn sich die Prüfungsbehörde im Verwaltungsverfahren zunächst darauf beschränke, lediglich die Bewertung bekannt zu geben. Die Prüfungsbehörde könne die Begründung im Beschwerdeverfahren nachreichen, sofern die betroffene Person in einem zweiten Schriftenwechsel dazu Stellung nehmen könne. ${ }^{18}$ In neueren Entscheiden äusserte sich das Bundesgericht eher dahingehend, dass im Verwaltungsverfahren zumindest eine mündliche Erläuterung notwendig ist und nur die schriftliche Begründung im Beschwerdeverfahren nachgeschoben werden darf. ${ }^{19}$

6 Das Bundesverwaltungsgericht lehnt seine Formulierungen zur Begründung von Prüfungsentscheiden in erster Linie an diejenigen der älteren Bundesgerichtsrechtsprechung an. So erwägt es jeweils zunächst, dass es genüge, der betroffenen Person kurz darzulegen, welche Lösungen von ihr erwartet worden seien und inwiefern ihre Antworten den Anforderungen nicht zu genügen vermocht hätten. Dieser Anspruch sei jedoch nicht

15 NIEHUES/FISCHER/JEREMIAS (Fn. 4), Rz. 706 und 710.

16 NIEHUES/FISCHER/JEREMIAS (Fn. 4), Rz. 707 f. und 713.

17 NIEHUES/FISCHER/JEREMIAS (Fn. 4), Rz. 712.

18 Urteil des Bundesgerichts 2C_1004/2017 vom 29. Mai 2018 E. 3.1; Urteil des Bundesgerichts 2D 65/2011 vom 2. April 2012 E. 5.1; sowie Urteil des Bundesgerichts 2P_23/2004 vom 13. August 2004 E. 2.2.

19 Urteil des Bundesgerichts 2D_29/2015 vom 27. November 2015 E. 2.2; Urteil des Bundesgerichts 2C_505/2019 vom 13. September 2019 E. 4.2.1; sowie Urteil des Bundesgerichts 2D_10/2019 vom 6. August 2019 E. 4.2. schon dann verletzt, wenn sich die Prüfungsbehörde vorerst darauf beschränke, die Bewertung bekannt zu geben. Sie könne die Begründung im Rechtsmittelverfahren nachliefern. Es reiche aus, wenn die betroffene Person in einem zweiten Schriftenwechsel dazu Stellung nehmen könne. ${ }^{20}$

Auch nach der Praxis des Verwaltungsgerichts des Kan- 7 tons Bern genügt es grundsätzlich, wenn die Prüfungsbehörde ihre Bewertung erst im Beschwerdeverfahren nachvollziehbar begründet. So verneinte es im Rahmen der Überprüfung einer Anwaltsprüfung im Jahr 2015 eine Verletzung des rechtlichen Gehörs. Die Beschwerdeführerin rügte u.a., dass die Bewertung der mündlichen Strafprozessrechtsprüfung nicht nachvollziehbar sei und die Notenbegründung fehle. Zunächst erwog das Verwaltungsgericht, dass mündliche Prüfungen je nach Gesprächsverlauf entsprechend vielgestaltig seien. Das Protokoll habe deshalb den Zweck, den Sachverhalt beweismässig zu sichern. Die Expertinnen müssten den Prüfungsverlauf zumindest grob nachzeichnen können. Die Begründung brauche sich nicht allein aus den Prüfungsprotokollen zu erschliessen. Es genüge, wenn die Nachvollziehbarkeit erst im Rechtsmittelverfahren durch die Stellungnahmen der Expertinnen hergestellt werde. ${ }^{21}$

In einem Urteil aus dem Jahr 2017 bekräftigte das Verwal- 8 tungsgericht des Kantons Bern diese Haltung. Auch in diesem Entscheid ging es um eine Anwaltsprüfung. Die Beschwerdeführerin rügte eine Verletzung der Begründungspflicht. Dem entgegnete das Verwaltungsgericht, dass es nach konstanter Rechtsprechung genüge, wenn die Nachvollziehbarkeit der Leistungsbewertung nachträglich, allenfalls erst im Rechtsmittelverfahren durch das Einholen von Stellungnahmen hergestellt werde. Die Anwaltsprüfungskommission habe sich im verwaltungsgerichtlichen Verfahren ausführlich zum Prüfungsergebnis geäussert, einschliesslich der Grenzfallthematik. Damit sei sie dem Anspruch auf eine ausreichende und nachvollziehbare Begründung nachgekommen. ${ }^{22}$

Das aktuellste Urteil des Verwaltungsgerichts des Kan- 9 tons Bern aus dem Jahr 2021 betraf das Nichtbestehen des schriftlichen Teils der Anwaltsprüfung. Das Verwaltungsgericht des Kantons Bern führte darin aus, dass die Begründung zumindest so abgefasst sein müsse, dass die Betroffenen die Verfügung sachgerecht anfechten könnten. Die Behörde komme dieser Pflicht nach, wenn sie

20 Urteil des Bundesverwaltungsgerichts B-822/2016 vom 24. August 2017 E. 5.2; Urteil des Bundesverwaltungsgerichts B-3020/2018 vom 12. Februar 2019 E. 4.3.

21 Urteil des Verwaltungsgerichts des Kantons Bern 100.2014.94U vom 30. März 2015 E. 5.1f.

22 Urteil des Verwaltungsgerichts des Kantons Bern 100.2016.231U vom 13. März 2017 E. 4.2. 
dem oder der Betroffenen kurz darlege, welche Lösungen bzw. Problemanalysen erwartet worden seien und inwiefern die Antworten den Anforderungen nicht genügt hätten. Die nähere Begründung der einzelnen Noten könne die Behörde auch nachträglich beibringen, sei es mündlich in einem Prüfungsgespräch, sei es mittels schriftlicher Stellungnahme im Rechtsmittelverfahren, sofern die betroffene Person dazu Stellung nehmen könne. ${ }^{23}$

In einem Urteil des Verwaltungsgerichts des Kantons Zürich aus dem Jahr 2011 ging es um Lizentiat II-Prüfungen der rechtswissenschaftlichen Fakultät der Universität Zürich. Das Verwaltungsgericht hielt fest, bei Prüfungsentscheiden komme die Behörde ihrer Begründungspflicht nach, wenn sie der betroffenen Person-allenfalls auch nur mündlich - kurz darlege, welche Lösungen von ihr erwartet worden seien und inwiefern ihre Antworten den Anforderungen nicht zu genügen vermocht hätten. Für die Begründung von Prüfungsentscheiden seien Noten grundsätzlich ein ausreichendes Mittel. Bei negativen Prüfungsentscheiden bestehe auf Gesuch hin ein Anspruch auf eine summarische, schriftliche Begründung, welche spätestens im Rechtsmittelverfahren nachzuliefern sei. Die betroffene Person müsse danach im zweiten Schriftenwechsel Gelegenheit zur Stellungnahme erhalten. Die Begründungspflicht gebiete es freilich nicht, dass die Behörde sämtliche Überlegungen zur Bewertung berücksichtige. Der Begründungspflicht sei Genüge getan, wenn sich die Behörde aufjene Gesichtspunkte beschränke, welche sie ohne Willkür als wesentlich betrachte. ${ }^{24}$ Schliesslich liess das Verwaltungsgericht offen, ob die Vorinstanz ihre Begründungspflicht verletzt hatte, da dieser Mangel geheilt worden wäre. Wegen eines fehlenden Bewertungsschemas wies das Verwaltungsgericht die Sache trotzdem an die Vorinstanz zurück und auferlegte der Beschwerdeführerin wegen ihrem teilweisen Unterliegen die Hälfte der Verfahrenskosten. ${ }^{25}$

11 In einem Urteil des Kantonsgerichts Luzern aus dem Jahr 2018 ging es sodann um eine praktische Führerprüfung. Das Kantonsgericht erwog zunächst, dass die Behörde ihrer Begründungspflicht nachkomme, wenn sie der betroffenen Person kurz darlege, inwiefern ihre Prüfungsleistungen den Anforderungen nicht zu genügen vermocht hätten. Der Anspruch auf Begründung sei nicht schon dann verletzt, wenn die Prüfungsbehörde sich vorerst darauf beschränke, die Bewertung bzw. das Nichtbestehen der Führerprüfung bekannt zu geben. Es genüge

\footnotetext{
23 Urteil des Verwaltungsgerichts des Kantons Bern 100.2019.426U vom 19. Februar 2021 E.3.2.

24 Urteil des Verwaltungsgerichts des Kantons Zürich VB.2010.00651 vom 13.Juli 2011 E. 3.1.

25 Urteil des Verwaltungsgerichts des Kantons Zürich VB.2010.00651 vom 13.Juli 2011 E. 3.2f., 9.3 und 10.
}

vielmehr, wenn sie die Begründung im Rechtsmittelverfahren liefere und die betroffene Person in einem zweiten Schriftenwechsel Stellung nehmen könne. ${ }^{26}$ Das Nichtbestehen wurde dem Prüfling unmittelbar nach der Führerprüfung erläutert. Der Prüfungsexperte liess dem Prüfling sodann eine Fehlerliste per E-Mail zukommen und erklärte ihm die Fehler auf dem Anrufbeantworter. Anschliessend verfügte das Strassenverkehrsamt das Nichtbestehen förmlich mit schriftlicher Begründung. Das Kantonsgericht qualifizierte diese Begründung als verfassungsmässig. ${ }^{27}$

In einem verwaltungsinternen Beschwerdeverfahren 12 vor der bernischen Erziehungsdirektion [ERZ] ${ }^{28} \mathrm{im} \mathrm{Jahr}$ 2017 ging es schliesslich um das Nichtbestehen der Diplomarbeit des Bildungsgangs Agrotechniker/-in HF. Die Beschwerdeinstanz erörterte, das Bundesgericht bejahe bei bildungsrechtlichen Leistungsbeurteilungen zumindest einen Anspruch auf summarische Begründung eines negativen Prüfungsentscheids. Die Begründungspflicht sei nicht schon dann verletzt, wenn sich die Prüfungsbehörde vorerst darauf beschränke, die Note bekannt zu geben. Es genüge, wenn die Prüfungsbehörde nach einer mündlichen Erläuterung die schriftliche Begründung im Rechtsmittelverfahren nachliefere und die betroffene Person in einem zweiten Schriftenwechsel umfassend dazu Stellung nehmen könne. Das Fehlen einer ausreichenden Begründung stelle sodann einen Rechtsfehler dar, welcher zur Aufhebung der Verfügung führe. ${ }^{29}$ Weiter hielt dieERZ fest, die vorliegende Begründung und ihr Aufbau würden insgesamt nicht ohne Weiteres nachvollziehbar erscheinen. Die Zuordnung der einzelnen Fehler und Mängel zu den jeweiligen Unterkriterien sei nicht eindeutig. Es bleibe daher unklar, wie die Unterkriterien bewertet worden seien. Weiter scheine der Aufbau des Bewertungsschemas nicht schlüssig. ${ }^{30}$ Die ERZ hiess die Beschwerde teilweise gut und wies die Sache an die Vorinstanz zurück. Obwohl die Beschwerdeführerin teilweise unterlag, sah die ERZ von einer Kostenauflage ab. ${ }^{31}$

26 Urteil des Kantonsgerichts Luzern 7H 17206 vom 20. März 2018 E.2.2.2.

27 Urteil des Kantonsgerichts Luzern 7H 17206 vom 20. März 2018 E.2.3.3 sowie 2.3.4.5.

28 Heute: Bildungs- und Kulturdirektion des Kantons Bern [BKD].

29 Zwischenentscheid der Erziehungsdirektion des Kantons Bern 4800.600.350.90/16 (758580) vom 17. Oktober 2017 E. 2.1 sowie BVR 2018 S.128f.

30 Zwischenentscheid der Erziehungsdirektion des Kantons Bern 4800.600.350.90/16 (758580) vom 17. Oktober 2017 E.2.2.1 sowie BVR 2018 S.128f.

31 Zwischenentscheid der Erziehungsdirektion des Kantons Bern 4800.600.350.90/16 (758580) vom 17. Oktober 2017 E. 3 sowie BVR 2018 S.128f. 


\section{Anforderungen an die Begründung} unbestritten, dass die Anforderungen an die Begründungsdichte im Prüfungsrecht variieren können. ${ }^{32}$ In diesem Sinne werden in einem ersten Schritt die allgemein gültigen Kriterien zu Prüfungsbegründungen behandelt. Sodann wird in einem zweiten Schritt auf die spezifischen Anforderungen eingegangen, welche bezüglich bestimmten Prüfungstypen gelten sollten.

\section{Allgemeines}

Einzelne Noten stellen gemäss h.L. und Rechtsprechung grundsätzlich Begründungselemente der endgültigen Verfügung (Gesamtbeurteilung) dar und sind demnach nicht selbständig anfechtbar. ${ }^{33}$ Trotzdem müssen meiner Meinung nach auch die nicht selbständig anfechtbaren Prüfungsbewertungen den nachfolgenden Anforderungen entsprechend begründet werden. Denn erst wenn der Prüfling die Bewertung dieser Teilnoten versteht, kann er die Gesamtbeurteilung nachvollziehen und diese sachgerecht anfechten. In der Volksschule und dem Gymnasium kommen die Prüfungsbehörden ihrer Begründungspflicht etwa nach, indem sie die Bewertung schriftlicher Prüfungen auf dem Prüfungsblatt kurz begründen und den Schülerinnen bei protokollierten mündlichen Prüfungen zumindest eine Prüfungsbesprechung anbieten. Ein Zeugnis, welches gestützt auf derart eröffnete Noten ergeht, ist meiner Meinung nach rechtsgenügend begründet.

spezialgesetzliche Regeln zur Begründung, welche über die Mindestgarantie von Art. 29 Abs. 2 BV hinausgehen, muss die Begründung zunächst diesen Anforderungen genügen. Weiter darf sich die Prüfungsbehörde bei ihrer Begründung auf diejenigen Momente beschränken, welche sie - ohne in Willkür zu verfallen als wesentlich erachtet. Angesprochen wird damit das Prinzip der antizipierten Beweiswürdigung. Zu Nebenpunkten, welche an der Bewertung ohnehin nichts ändern würden, braucht sie sich nicht zu äussern. ${ }^{34}$

Auch im Prüfungsrecht gilt sodann:Je schwerer der Eingriff in die Rechtsstellung der betroffenen Person wiegt, desto höher sind die Anforderungen an die Begründung.

32 Vgl. AUBERT (Fn. 5), S.144f.

33 Vgl. z.B. Urteil des Bundesverwaltungsgerichts B-2209/2006 vom 2.Juli 2007 E.1; Urteil des Bundesverwaltungsgerichts B-6256/2009 vom 14.Juni 2010 E. 7.1; Urteil des Verwaltungsgerichts des Kantons Bern 100.2019.335U vom 5. Mai 2020 E.1.2.2 sowie AUBERT (Fn. 5), S.73.

34 Vgl. dazu KIENER/KÄLIN/WYTTENBACH (Fn.3), S. 521; HÄFELIN/ MÜLLER/UHLMANN (Fn. 2), Rz.1071; sowie Urteil des Verwaltungsgerichts des Kantons Zürich VB.2010.00651 vom 13. Juli 2011 E.3.1.
Zudem ist die Begründungsdichte auch dann erhöht, wenn die Prüfungsbehörde ein grosses Ermessen geniesst. Daraus erhellt, dass ungenügende Noten grundsätzlich eingehender zu begründen sind.Je schwerer die Auswirkungen auf das berufliche oder anderweitige Fortkommen sind, desto besser muss die Leistungsbewertung begründet werden. Es leuchtet ein, dass an die Begründung einer Prüfungsbewertung in der 2. Klasse der Volksschule nicht dieselben Anforderungen gestellt werden können wie an diejenige im Rahmen einer Anwaltsprüfung.

\section{Schriftliche Prüfungen}

Bei schriftlichen Prüfungen kann unterschieden werden 17 zwischen Aufgaben mit offenem und geschlossenem Antwortformat. Textaufgaben sind z.B. der ersten und Multiple-Choice-Aufgaben der zweiten Kategorie zuzuordnen.

\section{a) Aufgaben mit geschlossenem Antwortformat}

Für Prüfungsaufgaben mit geschlossenem Antwortfor- 18 mat wie Multiple-Choice-Aufgaben und Prüfungen mit Ja-/Nein-Antwortkategorien liegt i.d.R. ein Lösungsraster vor. Weiter sind die Korrekturen meist leicht überprüfbar und dem Einfluss der Prüfungsexpertin weitgehend entzogen. Demnach gelten Prüfungen mitgeschlossenem Antwortformat als besonders objektiv. ${ }^{35}$

Bei solchen Aufgaben sollten keine hohen Anforderun- 19 gen an die Begründung gestellt werden. Meistens dürfte sich die Bewertung solcher Aufgaben bereits anhand des Lösungsrasters nachvollziehen lassen. Händigt die Prüfungsbehörde dem Prüfling somit das Lösungsraster aus, kommt sie ihrer Begründungspflichtgrundsätzlich rechtsgenügend nach. Dasselbe gilt, wenn die Prüfungsbehörde dem Prüfling die korrigierte Prüfung retourniert, auf welcher die erwarteten Antworten markiert sind. Dieses Vorgehen ist äusserst simpel und prozessökonomisch, weshalb die Prüfungsbewertung m.E. bereits im Verwaltungsverfahren schriftlich zu begründen ist.

\section{b) Aufgaben mit offenem Antwortformat}

Im Vergleich zu den ersterwähnten Aufgaben gelten sol- 20 che mit offenem Antwortformat als wenig objektiv. ${ }^{36}$ Die Antwortmöglichkeiten sind hier oft beinahe unbegrenzt, weshalb die Bewertung massgeblich von der jeweiligen Prüfungsexpertin abhängt. ${ }^{37}$ In diesem Sinne muss die Objektivität bei solchen Aufgabentypen durch

\footnotetext{
35 FLAVIO DI GIUSTO / CLAUDE MÜLLER WERDER / ANDREA REICHMUTH, Multiple-Choice-Aufgaben, 2018, S. 5; ZHAW, Multiple-Choice-Aufgaben, Teaching Guide for Higher \& Professional Education; sowie HELFRIED MOOSBRUGGER / AUGUSTIN KELAVA, Testtheorie und Fragebogenkonstruktion, 2. Aufl., Berlin/Heidelberg 2012, S. 8.
}

36 DI GIUSTO/MÜLLER WERDER/REICHMUTH (Fn. 35), S. 5.

37 AUBERT (Fn. 5), S. 8 . 
die Begründung und deren Nachvollziehbarkeit hergestellt werden. Infolgedessen erheischen offene Textaufgaben grundsätzlich eine eingehende Begründung der Bewertung. Nur bei leicht überprüfbaren Bewertungen (z.B. in unteren Volksschulklassen) kann die Bekanntgabe des Lösungsrasters bzw. der Notenskala eine rechtsgenügende Begründung darstellen. Weiter kann die Bewertung durch eine Mehrzahl von Prüfungsexpertinnen objektiviert werden (sog. Mehrprüferprinzip). ${ }^{38}$

Bei Prüfungsaufgaben mit offenen Antwortmöglichkeiten muss anhand der Begründung sodann die Validität der Bewertung nachvollzogen werden können. In der Testtheorie wird mit der Validität ausgedrückt, ob eine Prüfung das misst, was sie messen soll. ${ }^{39}$ Die Begründung soll dabei zunächst der Selbstkontrolle der Prüfungsbehörde dienen und verhindern, dass sich diese bei der Bewertung von sachfremden Aspekten leiten lässt. ${ }^{40}$ Weiter muss die Begründung sowohl dem Prüfling als auch der Beschwerdeinstanz erlauben, die Validität nachträglich zu überprüfen. Hat die Prüfungsbehörde bei der Bewertung sachfremde Kriterien berücksichtigt, ist die Prüfung bzw. ihre Bewertung nicht valid. Dies führt dazu, dass die Bewertung als nicht nachvollziehbar beurteilt werden muss.

Den hohen Anforderungen entsprechend sollte die Rechtsprechung bei solchen Prüfungsaufgaben bereits im Verwaltungsverfahren eine schriftliche Begründung verlangen. Dadurch wird die Begründung fixiert und der Prüfungsbehörde ist es in der Folge nicht mehr möglich, ihre Begründung im weiteren Beschwerdeverfahren beliebig zu ändern. ${ }^{41}$

\section{Mündliche Prüfungen}

23 Mündliche Prüfungsaufgaben ähneln testtheoretisch den schriftlichen Prüfungsaufgaben mit offenem Antwortformat. Wie das Verwaltungsgericht des Kantons Bern in einem Entscheid zu Recht festhielt, zeichnen sich mündliche Prüfungen durch ihre Vielgestaltigkeit aus. ${ }^{42}$ Auch bei diesen sind die Antwortmöglichkeiten beinahe unbegrenzt, weshalb die Anforderungen an die Begründung hoch sein sollten. Insofern kann grundsätzlich auf das unter Ziffer 2 Gesagte verwiesen werden.

38 AUBERT (Fn. 5), S.143; NIEHUES/FISCHER/JEREMIAS (Fn. 4), Rz. 547 39 Zum Begriff der Validität vgl. MOOSBRUGGER/KELAVA (Fn. 35), S.13. 40 AUBERT (Fn. 5), S. 144 .

$41 \mathrm{Zu}$ dieser schriftlichen «Konservierung» der Begründung bei schriftlichen Prüfungen vgl. NIEHUES/FISCHER/JEREMIAS (Fn.4), Rz.708.

42 Urteil des Verwaltungsgerichts des Kantons Bern 100.2014.94U vom 30. März 2015 E. 5.2.
Im Gegensatz zu schriftlichen Prüfungsaufgaben mit of- 24 fenem Antwortformat werden bei den mündlichen Aufgaben jedoch oft nicht einmal die gegebenen Antworten festgehalten. Die geltende Praxis verlangt gestützt auf Art. 29 Abs. 2 BV weder ein Prüfungsprotokoll noch eine Aufzeichnung. ${ }^{43}$ Entgegen der Ansicht von NIEHUES/FISCHER/JEREMIAS ${ }^{44}$ sprechen bei mündlichen Prüfungen demnach sogar mehr Gründe für eine schriftliche «Konservierung» der Begründung als bei schriftlichen Aufgaben. Teilweise Abhilfe schaffen könnte die Rechtsprechung, indem sie aus Art. 29 Abs. 2 BV neu auch im Prüfungsrecht einen Anspruch auf Aufzeichnung/Protokollierung der gegebenen Antworten ableiten würde. ${ }^{45} \mathrm{Da}$ bei muss m.E. nicht unbedingt ein Wortprotokoll erstellt werden. Es reicht aus, wenn die Expertinnen diejenigen Informationen festhalten, welche für die anschliessende Bewertung und deren Begründung massgebend sind.

Sodann wäre es zu begrüssen, wenn das Bundesgericht 25 die verwaltungsinternen Akten wie Handnotizen fortan dem Akteneinsichtsrecht unterstellen würde. Das Argument des Bundesgerichts, die interne Meinungsbildung solle nicht vollständig vor der Öffentlichkeit ausgebreitet werden, ${ }^{46}$ überzeugt gerade im Prüfungsrecht wenigzumal es sich kaum um sensible Informationen handelt. Es steht ausser Zweifel, dass den Handnotizen von Prüfungsexpertinnen Beweiseignung zukommt, bilden sie doch einen Zwischenschritt hin zur endgültigen Bewertung. Folglich sind sie grundsätzlich dazu geeignet, den Bewertungsvorgang zu veranschaulichen. Insofern das Bundesgericht diesen Aktenstücken zudem die Beweisbestimmung abspricht, unterliegt es einem Zirkelschluss, indem es den Begriff der verwaltungsinternen Akten erst geschaffen und ihnen dadurch die Beweisbestimmung abgesprochen hat. Schliesslich darf bezweifelt werden, dass der Verfassungsgeber derart geeignete Beweisstücke vollumfänglich vom Akteneinsichtsrecht ausnehmen wollte.

Liegt keine Aufzeichnung/Protokollierung vor, müsste 26 m.E. bereits im Verwaltungsverfahren eine summarische schriftliche Begründung verlangt werden, um der verfassungsrechtlichen Begründungspflicht Rechnung zu tragen. Dadurch kann verhindert werden, dass die Prüfungsbehörde ihre Bewertung infolge Zeitablaufs selbst

43 Vgl. z.B. Urteil des Bundesgerichts 2D_29/2015 vom 27. November 2015 E. 2.3; Urteil des Bundesgerichts 2P_23/2004 vom 13. August 2004 E. 2.4; sowie HÄFELIN/MÜLLER/UHLMANN (Fn. 2), Rz.1071.

44 NIEHUES/FISCHER/JEREMIAS (Fn. 4), Rz. 708 sowie 713.

45 Auch AUBERT (Fn. 5), S.143f., befürwortet eine Protokollierungspflicht gestützt auf Art. 29 Abs. 2 BV (Art. 4 Abs.1 aBV) bei mündlichen und praktischen Prüfungen. Zur aus Art. 29 Abs. 2 BV abgeleiteten Protokollierungspflicht in anderen Rechtsgebieten vgl. KIENER/ RÜTSCHE/KUHN (Fn.3), Rz.629.

46 Vgl. ARC HÄUSLER/ RETO FERRARI-VISCA, Das Recht auf Akteneinsicht im Verwaltungs- und Verwaltungsjustizverfahren, Jusletter vom 8. August 2011, S. 4; KIENER/RÜTSCHE/KUHN (Fn.3), Rz.633f. 
nicht mehr nachvollziehen kann. ${ }^{47}$ Darüber hinaus dient dieses Vorgehen der Transparenz gegenüber dem Prüfling und den Beschwerdebehörden. Wird der Prüfungsablauf nämlich protokolliert/aufgezeichnet oder dann zumindest in einer schriftlichen Begründung grob nachgezeichnet, ist es der Prüfungsbehörde nicht mehr ohne Weiteres möglich, ihn im Verlauf des Beschwerdeverfahrens abzuändern. Sodann weiss der Prüfling dadurch von Anfang an, von welchem Prüfungsablauf die Prüfungsbehörde ausgeht. ${ }^{48}$ Wurde der Prüfungsablauf bereits protokolliert/aufgezeichnet, kann eine mündliche Begründung im Verwaltungsverfahren aus prozessökonomischen Überlegungen ausreichen. ${ }^{49}$

\section{Praktische Prüfungen}

Praktische Prüfungen zeichnen sich dadurch aus, dass sie im Gegensatz zu den mündlichen und schriftlichen Prüfungen nicht Theoriefragen zum Gegenstand haben. Beispiele solcher Prüfungen sind die praktischen Teile von handwerklichen Lehrabschlussprüfungen oder praktische Führerprüfungen. Oftmals handelt es sich bei Prüfungen jedoch um Mischungen aus den hier angesprochenen Idealtypen. So kann eine praktische Führerprüfung neben dem praktischen Hauptteil u.a. mündliche Theoriefragen enthalten.

Praktische Prüfungsaufgaben ähneln in ihrer Vielgestaltigkeit den mündlichen Aufgaben, weshalb das dort Gesagte für sie grundsätzlich ebenfalls gilt. Trotzdem ist meiner Meinung nach der speziellen Natur von praktischen Prüfungen Rechnung zu tragen. Das nonverbale Verhalten im Rahmen von praktischen Prüfungen lässt sich oft weniger gut in Worte fassen. Bei praktischen Prüfungen ist eine ausführliche Protokollierung deshalb teilweise ausgeschlossen - so z.B. bei praktischen Führerprüfungen. In diesen Fällen genügt es meiner Ansicht nach deshalb, wenn der Fahrprüfungsexperte die relevanten Fahrfehler stichwortartig protokolliert. Damit der Prüfungsablauf fixiert werden kann, sollte die Praxis auch diesbezüglich eine zumindest rudimentäre Protokollierungspflicht aus Art. 29 Abs. 2 BV ableiten. ${ }^{50}$

47 So geschehen z.B. im Fall BVR 2012 S. 330 und 332.

48 Angesprochen wird mit dieser Argumentation die sog. Kompensation durch Verfahren. Durch erhöhte Anforderungen an das Verfahren kann die Prüfungsbewertung bis zu einem gewissen Mass objektiviert werden. Vgl. dazu AUBERT (Fn. 5), S.142.

49 Auch NIEHUES/FISCHER/JEREMIAS (Fn. 4), Rz.713, erachten eine mündliche Begründung bei mündlichen Prüfungen grundsätzlich für ausreichend. Offenbar wollen sie diese jedoch nur gewähren, wenn sie der Prüfling von sich aus verlangt. M.E. sollte die Prüfungsbehörde den Prüfling jedoch zumindest auf diese Möglichkeit hinweisen und explizit eine Prüfungsbesprechung anbieten.

50 Als vorbildliches Verhalten bezüglich der Begründung einer praktischen Prüfung im Verwaltungsverfahren kann hier etwa das Beispiel im Urteil des Kantonsgerichts Luzern 7H17 206 vom 20. März 2018 E. 2.3.3 angeführt werden. Der Fahrexperte und die Prüfungs-

\section{Zum sog. «Nachschieben» der Begründung}

Es erscheint sowohl in der deutschen als auch in der 29 schweizerischen Lehre und Rechtsprechung unbestritten, dass die Prüfungsbehörde ihre Begründung im Rechtsmittelverfahren ergänzen darf. Nicht einheitlich präsentiert sich die Rechtsprechung aber zur Frage, inwiefern im Verwaltungsverfahren zumindest eine summarische Bewertungsbegründung erforderlich ist.

In seinen älteren Entscheiden erwog das Bundesgericht 30 zwar teilweise ebenfalls, dass die Prüfungsbehörde ihrer Verpflichtung nachkomme, indem sie den Betroffenen - allenfalls nur mündlich - kurz darlege, welche Lösungen erwartet worden seien und inwiefern ihre Antworten den Anforderungen nicht zu genügen vermocht hätten. Es relativierte diese Aussage jedoch, indem es seine Standardformulierung - der Anspruch auf Begründung sei nicht schon dann verletzt, wenn die Prüfungsbehörde sich (vorerst) darauf beschränke, die Notenbewertung bekannt zu geben - hintanstellte. ${ }^{51}$ Wortlaut und Satzstellung der alten Entscheide sprechen demnach dafür, dass das Bundesgericht es als zulässig erachtete, im Verwaltungsverfahren lediglich die Bewertung bzw. Prüfungsnote bekannt zu geben. Im Jahr 2015 fällte das Bundesgericht offenbar erstmals einen Entscheid, in welchem es die Standardformulierung voranstellte und anschliessend bereits im Verwaltungsverfahren explizit eine mündliche Erläuterung verlangte. ${ }^{52}$ In einem Entscheid aus dem Jahr 2018 wählte das Bundesgericht jedoch wieder die alte Satzstellung und erwähnte, dass die Bewertung bzw. Note im Verwaltungsverfahren eine ausreichende Begründung darstelle. ${ }^{53}$ Demgegenüber sprechen die neusten Bundesgerichtsentscheide wiederum eher dafür, dass die Prüfungsbehörde ihre Bewertung bereits im Verwaltungsverfahren summarisch begründen muss. ${ }^{54}$

Die bundesgerichtliche Rechtsprechung präsentiert 31 sich insofern missverständlich bzw. widersprüchlich. Ohnehin ist nicht klar, ob das Bundesgericht mit seinen neueren Urteilen eine Praxisänderung vornehmen wollte oder ob es sich um eine redaktionelle Ungenauigkeit handelt. Die Rechtsprechung betrachtet zudem einzelne Noten einer Gesamtbeurteilung grundsätzlich als

behörde haben dem Prüfling das Nichtbestehen ausführlich und sowohl mündlich als auch schriftlich erläutert (vgl. auch die Zusammenfassung des Urteils unter III.).

51 Urteil des Bundesgerichts 2D_65/2011 vom 2. April 2012 E. 5.1.

52 Urteil des Bundesgerichts 2D_29/2015 vom 27. November 2015E. 2.2. 53 Urteil des Bundesgerichts 2C_1004/2017 vom 29. Mai 2018 E. 3.1.

54 Urteil des Bundesgerichts 2C_505/2019 vom 13. September 2019 E. 4.2.1; Urteil des Bundesgerichts 2D_10/2019 vom 6. August 2019 E.4.2. 
Begründungselemente. ${ }^{55}$ Dennoch kann auch bei einer Gesamtbeurteilung, welche aus mehreren Einzelnoten besteht, nicht davon ausgegangen werden, dass die simple Bekanntgabe der Teilnoten den Mindestanforderungen von Art. 29 Abs. 2 BV im Verwaltungsverfahren genügt. Denn die Teilnoten sind nicht aus sich selbst verständlich. Sie erlauben es dem Prüfling nicht, die Gesamtbeurteilung sachgerecht anzufechten, geschweige denn die Prozesschancen einzuschätzen.

Die widersprüchlichen Formulierungen des Bundesgerichts beeinflussen auch die Rechtsprechung der unteren Beschwerdeinstanzen. Das Bundesverwaltungsgericht lässt es in seiner stetigen Rechtsprechung genügen, wenn die Prüfungsbehörde im Verwaltungsverfahren lediglich die Noten bzw. Bewertungen bekannt gibt. 56 Die gleiche Auffassung vertrat auch das Kantonsgericht Luzern in einem Entscheid aus dem Jahr 2018 und das Verwaltungsgericht des Kantons Zürich in einem Entscheid aus dem Jahr 2011. ${ }^{57}$ Dagegen erachtete die ERZ des Kantons Bern in ihrem Entscheid aus dem Jahr 2017 eine zumindest summarische Begründung als notwendig. ${ }^{58}$ Auch das Verwaltungsgericht des Kantons Bern fordert in seinem aktuellsten Urteil aus dem Jahr 2021 zumindest eine summarische Begründung im Verwaltungsverfahren, sodass der Prüfling die Verfügung sachgerecht anfechten kann. ${ }^{59}$

33 Meiner Meinung nach ist der Haltung des Bundesgerichts in seinen neueren Urteilen und derjenigen des Verwaltungsgerichts des Kantons Bern sowie der damaligen ERZ des Kantons Bern zu folgen. Es ist nicht einzusehen, weshalb es der Prüfungsbehörde aus prozessökonomischen Gründen nicht möglich sein sollte, zumindest eine summarische Begründung im Verwaltungsverfahren zu präsentieren. Da die Prüfungsbehörde diese Begründung im Beschwerdeverfahren ohnehin nachliefern muss, relativiert sich das Argument der Prozessökonomie. ${ }^{60}$ Erst durch diese Begründung ist es dem Prüfling ansatzweise möglich die Gedanken nachzuvollziehen, von denen sich die Expertinnen haben leiten lassen. Es widerspricht prozessökonomischen Überlegungen, wenn der Prüfling

\footnotetext{
55 Vgl. z.B. Urteil des Bundesverwaltungsgerichts B-2209/2006 vom 2.Juli 2007 E. 1; Urteil des Bundesverwaltungsgerichts B-6256/2009 vom 14.Juni 2010 E. 7.1.

56 Vgl. u.a. Urteil des Bundesverwaltungsgerichts B-3020/2018 vom 12. Februar 2019 E. 4.3.

57 Urteil des Kantonsgerichts Luzern 7H17 206 vom 20. März 2018 E. 2.2.2; Urteil des Verwaltungsgerichts des Kantons Zürich VB.2010.00651 vom 13.Juli 2011 E.3.1.

58 Zwischenentscheid der Erziehungsdirektion des Kantons Bern 4800.600.350.90/16 (758580) vom 17. Oktober 2017 E. 2.1, BVR 2018 S. $128 \mathrm{f}$.

59 Urteil des Verwaltungsgerichts des Kantons Bern 100.2019.426U vom 19. Februar 2021 E.3.2.

60 Vgl. KNEUBÜHLER (Fn.3), S. 108.
}

ein Beschwerdeverfahren anstrengen muss, um eine Begründung zu erhalten. ${ }^{61}$

Demnach sollte das Bundesgericht seine Rechtsprechung 34 meiner Meinung nach dahingehend ändern bzw. präzisieren, dass Prüfungsbewertungen bereits im Verwaltungsverfahren zumindest summarisch begründet werden müssen. Beschränkt sich die Prüfungsbehörde dagegen darauf, lediglich die Note bzw. Bewertung bekannt zu geben, liegt m.E. eine Verletzung des rechtlichen Gehörs vor. Diese kann vor einer Beschwerdeinstanz mit derselben Kognition grundsätzlich geheilt werden, wenn die Prüfungsbehörde im Rechtsmittelverfahren eine nachvollziehbare Begründung nachschiebt. ${ }^{62}$ Trotzdem dürfen dem Prüfling aus dieser Gehörsverletzung keine Nachteile erwachsen, weshalb auf eine Kostenauflage zu verzichten ist. ${ }^{63}$

Weiter kann eine mündliche Begründung bei mündli- 35 chen und praktischen Prüfungen im Verwaltungsverfahren meiner Meinung nach nur dann genügen, wenn der Prüfungsablauf zumindest rudimentär fixiert worden ist - durch ein Protokoll oder eine Aufzeichnung. Dadurch wird verhindert, dass die Prüfungsexpertinnen den Prüfungsablauf selbst nicht mehr rekonstruieren können. Wie bereits unter III.3 dargetan, dient dieses Vorgehen zudem der Transparenz.

Sodann spricht auch der Grundsatz von Treu und Glau- 36 ben nach Art. 5 Abs. 3 BV für eine Begründungspflicht im Verwaltungsverfahren. Verzichtet die Prüfungsbehörde im Verwaltungsverfahren darauf, dem Prüfling auch nur ansatzweise darzulegen, weshalb er nicht bestanden hat, kann dieser seine Prozesschancen nicht abschätzen. Stattdessen ist er gezwungen ein Beschwerdeverfahren anzustrengen, damit die Prüfungsbehörde ihre Karten offenlegt. Infolgedessen erscheint es als treuwidrig, wenn der Staat ihm dafür Verfahrenskosten auferlegt.

Etwas anderes kann nur dann gelten, wenn die fehlende 37 Begründung im Verwaltungsverfahren durch den Prüfling verschuldet ist. Dies ist etwa dann der Fall, wenn der Prüfling die Teilnahme an einer Prüfungsbesprechung

61 Vgl. SUTTER (Fn.3), Art. 29 Rz. 25; das anfängliche Weglassen der Begründung im Verwaltungsverfahren provoziert das Ergreifen von Rechtsmitteln geradezu und führt zudem zu einem zweiten Schriftenwechsel im Beschwerdeverfahren.

62 Vgl. KNEUBÜHLER (Fn.3), S.104.

63 Als positives Beispiel kann hier etwa das Urteil des Verwaltungsgerichts des Kantons Bern 100.2015.177U vom 5. November 2015 E. 6 angeführt werden. In diesem Urteil verzichtete das Verwaltungsgericht des Kantons Bern auf eine Kostenauflage an den unterliegenden Beschwerdeführer, weil die Prüfungsbehörde substanzielle Ausführungen - insbesondere zu ihrer Grenzfallpraxis - erst in der Beschwerdevernehmlassung vorgebracht hatte. Durch dieses Vorgehen des Verwaltungsgerichts sind dem Beschwerdeführer aus der Gehörsverletzung keine Nachteile erwachsen. 
ablehnt. ${ }^{64}$ In diesem Fall verhält sich der Prüfling selbst treuwidrig und es liegt keine Verletzung von Art. 29 Abs. 2 BV vor. Infolgedessen können ihm bei Abweisung der Beschwerde grundsätzlich die vollen Kosten auferlegt werden.

\section{Rechtsfolgen der verletzten Begründungspflicht}

Der Anspruch auf rechtliches Gehör und damit auch der Teilgehalt der Begründungspflicht ist formeller Natur. Dessen Verletzung führt grundsätzlich zur Aufhebung des Prüfungsentscheids. ${ }^{65}$ Wie bereits erwähnt, lässt die Rechtsprechung die Heilung der verletzten Begründungspflicht bzw. das Nachschieben der Begründung jedoch grosszügig zu. Schiebt die Prüfungsbehörde eine nachvollziehbare Begründung vor einer Beschwerdeinstanz mit gleicher Kognition nach, gilt die Verletzung grundsätzlich als geheilt. ${ }^{66}$ Dadurch wird der Rüge der mangelnden Nachvollziehbarkeit der Boden entzogen und die Beschwerde erscheint in diesem Punkt fortan unbegründet. Infolgedessen ist sie abzuweisen - vorbehältlich anderer begründeter Rügen. Da dem Beschwerdeführer aus der anfänglichen Gehörsverletzung jedoch keine Nachteile erwachsen dürfen, muss der Staat die Kosten tragen. Aus diesem Grund ist es meiner Meinung nach einer Beschwerdeinstanz untersagt, die Frage der Gehörsverletzung offenzulassen und dem Beschwerdeführer trotzdem Kosten aufzuerlegen. ${ }^{67}$ Dadurch begeht die Beschwerdeinstanz eine formelle Rechtsverweigerung.

64 Vgl. dazu etwa BVR 2016 S. 450 E.3.3.

65 KIENER/RÜTSCHE/KUHN (Fn.3), Rz.272; SUTTER (Fn.3), Art. 29 Rz. 17; WIEDERKEHR (Fn. 5), S. 502.

66 WIEDERKEHR (Fn. 5), S. 502; KNEUBÜHLER (Fn. 3), S.104; SUTTER (Fn.3), Art.29 Rz. 25.

67 Ein schlechtes Beispiel in dieser Hinsicht ist etwa das Urteil des Verwaltungsgerichts des Kantons Zürich VB.2010.00651 vom 13.Juli 2011. Hier hat das Verwaltungsgericht das Vorliegen einer Gehörsverletzung offengelassen mit der Begründung, eine solche könne ohnehin geheilt werden (E.3.3). Trotzdem hat es der Beschwerdeführerin Kosten auferlegt (E.10).
Kann die Verletzung der Begründungspflicht nicht geheilt 39 werden, erscheint die Bewertung nicht nachvollziehbar. Demnach ist die Beschwerde gutzuheissen und der Prüfungsentscheid ist aufzuheben. Da es der Beschwerdebehörde aufgrund der mangelnden Nachvollziehbarkeit nicht möglich ist eine Neubewertung vorzunehmen, kann sie lediglich kassatorisch entscheiden und die Sache zur Neubewertung zurückweisen oder dem Beschwerdeführer eine Wiederholungsmöglichkeit gewähren. ${ }^{68}$

\section{Fazit}

Wie eben aufgezeigt, stellen verschiedene Prüfungstypen 40 unterschiedliche Anforderungen an ihre Begründung. Grundsätzlich sollten Bewertungen schriftlicher Prüfungen bereits im Verwaltungsverfahren schriftlich begründet werden - allenfalls nur summarisch. Sodann kann m.E. eine mündliche Begründung bei mündlichen und praktischen Prüfungen im Verwaltungsverfahren genügen, wenn der Prüfungsablauf anderweitig festgehalten wurde. Dementsprechend sollte die Rechtsprechung aus Art. 29 Abs. 2 BV eine Protokollierungs-/Aufzeichnungspflicht ableiten. Weiter sollte das Bundesgericht seine Rechtsprechung zu den verwaltungsinternen Akten ändern. Handnotizen von Prüfungsexpertinnen und ähnliche Aktenstücke sind als Beweismittel geeignet, weshalb sie offengelegt werden sollten.

Zurzeit ist unklar, ob die bundesgerichtliche Rechtspre- 41 chung im Verwaltungsverfahren eine über die schlichte Notenbekanntgabe hinausgehende Begründung verlangt. Das Bundesgericht könnte Klarheit schaffen, indem es seine Rechtsprechung im oben genannten Sinn präzisiert und eine solche explizit verlangt.

68 Vgl. z.B. Zwischenentscheid der Erziehungsdirektion des Kantons Bern 4800.600.350.90/16 (758580) vom 17. Oktober 2017 E.2.2.2, BVR 2018 S.128f. 